\title{
ORIGINAL
}

\section{Propriedades psicométricas da versão portuguesa da Nova Escala de Satisfação Sexual - versão curta}

s Q2 Pedro Santos Pechorro $\Lambda_{\Lambda}^{a, *}$, Patrícia Monteiro Pascoall ${ }_{\Lambda}^{b, c}$, Saul Neves Jesus $s_{\Lambda}^{a}$, 6 Ana Isabel Almeida ${ }_{\Lambda}^{d}$, $\Lambda_{\Lambda}$ Catarina Soares Figueiredo ${ }_{\Lambda}^{d}$ e Rui Xavier Vieira ${ }_{\Lambda}{ }^{2}$

7 Q3 a Centro de Investigação sobre Espaço e Organizações, Universidade do Algarve, Faro, Portugal

${ }^{\mathrm{b}}$ Faculdade de Psicologia e Ciências da Educação, Universidade do Porto, Porto, Portugal

c Escola de Psicologia e Ciências da Vida, Universidade Lusófona de Humanidades e Tecnologias, Lisboa, Portugal

d Instituto Universitário da Maia, Maia, Portugal

e Faculdade de Medicina, Universidade de Lisboa, Lisboa, Portugal

Recebido a 18 de janeiro de 2015; aceite a 23 de abril de 2016

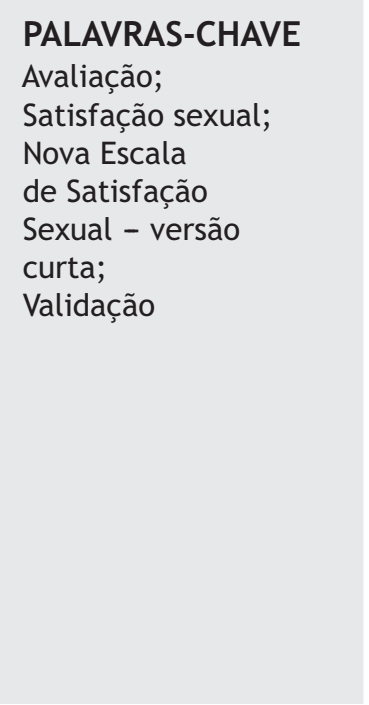

\begin{abstract}
Resumo
Introdução: A satisfação sexual constitui atualmente um constructo essencial no campo do estudo da sexualidade humana.

Objetivo: A presente investigação teve como objetivo proceder à validação da versão portuguesa da Nova Escala de Satisfação Sexual - versão curta (NSSS-S), instrumento em formato curto que avalia a satisfação sexual em homens e mulheres.

Material e métodos: Recorreu-se a um total de 298 participantes de ambos os sexos, os quais preencheram o questionário com a tradução para português da NSSS-S.

Resultados: Foram demonstradas as principais propriedades psicométricas da validação da NSSS-S, das quais se destacaram a estrutura fatorial bidimensional e o alfa de Cronbach $(\geq 0,89)$. Discussão: A NSSS-S revelou ter a estrutura bidimensional da NSSS original e obtiveram-se valores bons a nível de consistência interna, de validade convergente, de validade divergente e de validade concorrente.

Conclusões: As boas propriedades psicométricas encontradas justificam e reforçam a recomendação de utilização da NSSS-S na população portuguesa.

(c) 2016 Asociación Española de Andrología, Medicina Sexual y Reproductiva. Publicado por Elsevier España, S.L.U. Todos os direitos reservados.
\end{abstract}

\footnotetext{
* Autor para correspondência.

Correio eletrónico: ppechorro@gmail.com (P. Santos Pechorro).
} 


\section{KEYWORDS}

Assessment;

Sexual satisfaction;

New Sexual

Satisfaction

Scale-Short format;

Validation
Psychometric properties of the Portuguese version of the New Sexual Satisfaction Scale-Short

\begin{abstract}
Introduction: Sexual satisfaction is an essential construct in the study of human sexuality Objective: The aim of the present study was to validate the Portuguese version of the New Sexual Satisfaction Scale-Short (NSSS-S), a short form scale that assesses sexual satisfaction among men and women.

Material and methods: A total of 298 participants completed the Portuguese version of the NSSS-S.

Results: The main psychometric properties of the Portuguese version of the NSSS-S were assessed, most importantly the two-factor structure and Cronbach's alpha $(\geq 0.89)$.

Discussion: The NSSS-S revealed the bidimensional structure of the original NSSS and good values were obtained in terms of internal consistency, convergent validity, divergent validity and concurrent validity.

Conclusions: The use of the Portuguese version of the NSSS-S is justified and reinforced since it has sound psychometric properties.

(C) 2016 Asociación Española de Andrología, Medicina Sexual y Reproductiva. Published by Elsevier España, S.L.U. All rights reserved.
\end{abstract}

\section{Introdução}

A satisfação sexual, definida como «[...] uma resposta afetiva que resulta da avaliação subjetiva das dimensões positivas e negativas relacionadas com a relação sexual» ${ }^{1}$, é um objetivo importante para a atividade sexual e um indicador de saúde sexual ${ }^{2,3}$. Além de estar intimamente associada, a nível individual, à saúde e bem-estar e ao ajustamento emocional ${ }^{4}$, a satisfação sexual está ainda fortemente associada à qualidade, ajustamento e satisfação relacionais $^{5}$.

A demonstrada importância da satisfação sexual no bem-estar sustenta o seu papel privilegiado como indicador da vivência positiva da sexualidade, mas também a sua centralidade no estudo do bem-estar geral e da conjugalidade ${ }^{6}$. Apesar da investigação na área da satisfação sexual ter vindo a crescer nos últimos anos, reforçando a centralidade desta dimensão, tem sido salientado que a avaliação deste constructo é problemática ${ }^{7}$, uma vez que a satisfação sexual tem sido estudada com medidas muito diferentes, umas acentuando a dimensão conflitual dentro da relação ${ }^{8}$; outras acentuando a experiência clinicamente significativa de disfunção sexual ${ }^{9}$; outras ainda acentuando a dimensão relacional da sexualidade, em detrimento da experiência individual ${ }^{10}$.

A existência de uma considerável diversidade de medidas, que dificulta a comparabilidade entre os diversos estudos, aliada à quase inexistência de modelos empiricamente validados de explicação do constructo, tornam a seleção do melhor instrumento para medir a satisfação sexual problemática. Recentemente, num estudo comparativo de diversas medidas que avaliam a satisfação sexual e que são frequentemente utilizadas na literatura ${ }^{11}$, concluiu-se que a versão reduzida da Nova Escala de Satisfação Sexual (New Sexual Satisfaction Scale - Short [NSSS-S] ${ }^{7}$ ) demonstrou ter um comportamento, do ponto de vista psicométrico, estável, o que, comparativamente a outras medidas, justifica a sua seleção como melhor medida de satisfação sexual para utilização na investigação científica. Adicionalmente, do ponto de vista do conteúdo dos itens e sua organização teórica, a NSSS-S apresenta-se concetualmente como uma medida adequada à operacionalização de satisfação sexual ${ }^{12}$. Assim, este instrumento alia a adequação teórica e concetual ao constructo com o comportamento psicométrico estável e ainda a vantagem comum a instrumentos de medida curtos, i.e., a associação à maior rapidez na resposta aos protocolos de investigação, muitas vezes à custa de apresentar fidelidade e validade mais baixas que as escalas originais ${ }^{13}$.

A NSSS-S é a versão reduzida da Nova Escala de Satisfação Sexual (New Sexual Satisfaction Scale [NSSS] ${ }^{7}$ ). A NSSS original é constituída por 20 itens que medem a avaliação subjetiva da experiência sexual pessoal (centração no eu) e a avaliação da experiência interpessoal (centração no parceiro e na atividade sexual). Tanto na NSSS como na NSSS-S, os itens utilizam uma escala ordinal de 5 pontos (de 1 = nada satisfeito a 5 = extremamente satisfeito) e são somados para obter um valor total. Quanto mais alto este valor, maior a satisfação sexual. Contudo, a versão reduzida, constituída por 12 itens, é descrita com unidimensional e apresenta, ao contrário do que é habitual com a maioria das versões reduzidas de instrumentos de medida, validade e fidelidade semelhantes à escala original.

Nos estudos desenvolvidos com amostras universitárias e comunitárias, quer nos Estados Unidos da América quer na Croácia, a fidelidade da escala foi avaliada através do alfa de Cronbach que apresentou valores indicadores de boa consistência interna $(\alpha=0,90$ e 0,93$)$ e estabilidade temporal $(r=0,72 \text { a } 0,84)^{7}$. Relativamente aos estudos de validade, a NSSS-S apresentou validade convergente e validade divergente consideradas satisfatórias, assim como capacidade de descriminar corretamente $63 \%$ de casos clínicos e $80 \%$ não 
clínicos ${ }^{14}$. Esta medida foi recentemente usada num estudo junto da população masculina heterossexual em relação de compromisso estabelecendo que existe uma associação entre a intimidade emocional e a satisfação sexual ${ }^{15}$, um resultado consistente com outra investigação feita com uma amostra clínica ${ }^{16}$.

A presente investigação refere-se ao processo de validação da NSSS-S em uma amostra comunitária da população portuguesa. A satisfação sexual é um indicador importante de saúde sexual que apresenta uma relação forte com a satisfação relacional e ajustamento conjugal, pelo que o seu estudo é importante para a avaliação e promoção da saúde individual e relacional. Dado que em Portugal são raros os casos de medidas no campo da sexualidade validadas com sucesso (e.g., IIEF- $5^{17}$ ), a escolha da NSSS-S pareceu-nos particularmente enquadrada, de forma a fomentar o desenvolvimento da investigação em satisfação sexual.

\section{Objetivos}

Em Portugal, e também a nível internacional, existe a necessidade de proceder à validação de instrumentos psicométricos relativos ao campo da sexualidade humana que demonstrem ser válidos e fiáveis. 0 objetivo do presente artigo consistiu em proceder à adaptação portuguesa da NSSS-S, replicando alguns dos procedimentos métricos utilizados originalmente na construção desta escala e efetuando alguns novos. Pretende-se, assim, fundamentar a utilização da NSSS-S a nível de investigação e de clínica em Portugal.

\section{Material e métodos}

\section{Participantes}

A amostra normativa de conveniência da população geral foi constituída por 298 participantes (média = 30,57 anos; desvio-padrão $=9,57$ anos; amplitude $=18-63$ anos) residentes em meio urbano. Desse total de participantes, $51 \%$ eram mulheres e $49 \%$ eram homens. Em relação ao estado civil, $56,4 \%$ eram solteiros, $26,5 \%$ eram casados/em união de facto e 4,8\% eram divorciados/separados, e $12,3 \%$ tinham outro estado civil (e.g., viúvo) ou não responderam. Relativamente à escolaridade, 3,7\% tinham o ensino básico, $17,6 \%$ o ensino secundário, $77,1 \%$ tinham ensino superior e $1,6 \%$ não responderam. Em termos de números de parceiros sexuais atuais, 92,6\% afirmaram ter apenas um parceiro sexual, 1,6\% afirmaram ter mais de um parceiro sexual e $5,8 \%$ não responderam. No que diz respeito à frequência de atividade sexual, $22,9 \%$ referiram $1-2$ vezes por mês, $61,7 \%$ referiram $1-3$ vezes por semana, $10,6 \%$ referiram $4-6$ vezes por semana, $1,1 \%$ mais de 7 vezes por semana e $3,7 \%$ não responderam.

\section{Instrumentos}

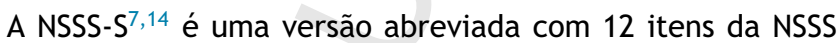
já validada em Portugal $^{18}$, que na sua forma original é composta por 20 itens. A NSSS original possui uma estrutura fatorial bidimensional constituída por uma subescala de centração no eu (subescala A: itens 2, 3, 5, 6, 8 e 10) e uma subescala de centração no parceiro e na atividade sexual (subescala B: itens 11, 12, 14, 17, 19 e 20), mas a NSSS-S é descrita como unidimensional. Os itens que constituem a NSSS e a NSSS-S são ordinais de 5 pontos (de 1 = nada satisfeito a 5 = totalmente satisfeito). A pontuação total da NSSS-S é obtida pela soma das pontuações de todos itens. Valores altos na pontuação correspondem a níveis altos de satisfação sexual. Pode ser utilizada com homens e mulheres, tendo sido desenvolvida a partir de amostras comunitárias, amostras clínicas e amostras de estudantes universitários. A NSSS-S tem demonstrado possuir boas propriedades psicométricas a nível de validade e fiabilidade.

A Escala de Busca de Sensações Sexuais (Sexual Sensation Seeking Scale $[\text { SSSS }]^{19,20}$ ) é uma escala unidimensional com 10 itens concebida para avaliar a busca de sensações sexuais - definida como a necessidade de ter experiências sexuais novas e variadas, e de correr riscos físicos e sociais com o objetivo de aumentar as sensações sexuais. Os itens que constituem a SSSS são ordinais de 4 pontos (de $1=$ discordo totalmente a 4=concordo totalmente). A SSSS pode ser utilizada com homens e mulheres, adultos ou adolescentes, tendo sido desenvolvida a partir de amostras comunitárias, amostras clínicas e amostras escolares/universitárias. A SSSS tem demonstrado possuir boas propriedades psicométricas a nível de validade e fiabilidade. Valores altos na pontuação da escala correspondem a níveis altos de busca de sensações sexuais. A versão portuguesa da SSSS $^{21}$ foi utilizada na presente investigação para efetuar a validade convergente, tendo a consistência interna por alfa de Cronbach obtida sido 0,74.

A Escala de Aborrecimento Sexual (Sexual Boredom Scale $[\mathrm{SBS}]^{22}$ ) é uma medida unidimensional com 18 itens que avalia o aborrecimento sexual, i.e., a tendência de determinada pessoa em sentir-se aborrecida com os diversos aspetos da sua vida sexual. Os itens que constituem a SBS são ordinais de 7 pontos (de 1 =discordo totalmente a 7 = concordo totalmente). A SBS pode ser utilizada com homens e mulheres, tendo sido originalmente desenvolvida a partir de diversas amostras de estudantes universitários. A escala SBS tem demonstrado possuir boas propriedades psicométricas a nível de validade e fiabilidade. Valores altos na pontuação da escala correspondem a níveis mais altos de aborrecimento sexual. A versão portuguesa da SBS $^{23}$ foi utilizada na presente investigação para efetuar a validade divergente, tendo a consistência interna por alfa de Cronbach obtida sido 0,93.

Foi construído adicionalmente um questionário sociodemográfico, através do qual se pretendeu recolher informação acerca de cada participante, nomeadamente: idade, sexo, nacionalidade, escolaridade, profissão, estado civil, duração do relacionamento atual, número de parceiros sexuais, frequência de atividade sexual (codificada como item ordinal de 5 pontos) e satisfação com vida sexual (codificada como item ordinal de 5 pontos).

\section{Procedimentos}

Foi contatado o primeiro autor da NSSS-S, nomeadamente Aleksandar Stulhofer, no sentido de obter permissão para se 
efetuar a validação portuguesa da NSSS-S, tendo este respondido que concedia a permissão solicitada. Inicialmente, foi feita uma tradução do instrumento com a colaboração de um tradutor-especialista. Os itens foram traduzidos literalmente sempre que o seu significado em português o permitisse, mas quando tal não era possível optou-se por uma tradução menos literal que captasse o sentido do item original $^{24}$. Foram de seguida feitas algumas aplicações experimentais, empregando-se para tal um contexto de grupo de foco e um contexto individual. A partir destas aplicações, evidenciou a necessidade de proceder a algumas pequenas correções adicionais à tradução, de forma a facilitar a leitura por parte dos participantes com níveis de escolaridade mais baixos, tendo-se chegado assim à versão final da escala.

Através do termo de consentimento informado que precedia o questionário, todos os participantes foram informados que o objetivo era fazer a validação da NSSS-S para a população portuguesa, que apenas os investigadores teriam acesso às respostas dos instrumentos de avaliação e que a participação era voluntária. Informou-se ainda que esta pesquisa tinha um caráter académico e que os investigadores não estariam interessados em resultados individuais, mas sim na análise estatística que abrangeria todas as respostas recolhidas. Recrutaram-se os participantes constituintes da amostra normativa de conveniência da população geral em instituições de ensino superior (Instituto Universitário da Maia, Universidade Lusófona de Humanidades e Tecnologias) que incluíram alunos e funcionários. Após a recolha dos dados, procedeu-se à seleção dos questionários que cumpriam os critérios da investigação, tendo sido excluídos os que não os cumpriam. Os critérios mínimos estabelecidos na inclusão dos participantes na presente investigação foram: ter nacionalidade portuguesa, ser maior de idade (18 ou mais anos de idade) e ter um relacionamento sexual há pelo menos 3 meses.

Os dados obtidos foram inseridos e tratados no software IBM SPSS v22 ${ }^{25}$. Após a inserção dos dados, foi recolhida uma amostra de cerca de $30 \%$ dos questionários inseridos na base de dados de forma a avaliar a qualidade de inserção, que veio a ser considerada boa dada a quase inexistência de erros de inserção. No tratamento de dados propriamente dito utilizando o SPSS, recorreu-se à análise de componentes principais (ACP), coeficiente alfa de Cronbach e correlações de Pearson, além de estatísticas descritivas (e.g., médias, desvios-padrão, percentis).

Recorreu-se também ao software EQS $6.2^{26}$ para efetuar a análise fatorial confirmatória (AFC). Os índices de ajustamento calculados incluíram: qui-quadrado de Satorra-Bentler/graus de liberdade, Comparative Ft Index (CFI), Incremental Fit Index (IFI), Root Mean Square Error of Approximation (RMSEA). Um qui-quadrado/graus de liberdade $<5$ é considerado adequado, $\leq 2$ é considerado bom e valores $=1$ são considerados muito bons. $C F I \geq 0,90$ e RMSEA $\leq 0,10$ indicam um ajustamento adequado, enquanto $\mathrm{CFI} \geq 0,95$ e RMSEA $\leq 0,06$ indicam um ajustamento bom ${ }^{27}$. Um IFI $\geq 0,90$ é considerado aceitável e um IFI $\geq 0,95$ é considerado bom. Em caso de necessidade, seriam utilizados índices de modificação para melhorar o ajustamento. A análise foi efetuada diretamente nos itens e somente valores com saturação $\geq 0,50$ foram considerados. Optou-se pela utilização de correlações policóricas, com métodos robustos de estimação nos itens ordinais, porque estas proporcionam melhores resultados ${ }^{27,28}$.

\section{Resultados}

O primeiro passo na validação da NSSS-S envolveu o cálculo das correlações item-total corrigidas (CITC), das saturações por ACP e das saturações por AFC para a amostra normativa e para a amostra clínica. Como a NSSS original é tida como uma medida bidimensional, entendeu-se verificar se esta estrutura também estaria presente na NSSS-S. Para tal, forçou-se a extração de 2 componentes empregando rotação Varimax, tendo o teste de Kaiser-Meyer-Olkin (KMO) indicado um valor de 0,92 e o teste de Bartlett sido estatisticamente significativo $\left(\chi^{2}=1.620,89 ; p \leq 0,001\right)$. Os critérios do Eigenvalue e do Scree plot foram compatíveis com a existência duma estrutura bidimensional, que se veio a verificar ser responsável por $69,01 \%$ da variância total. Apesar de haver 2 itens que saturaram de forma cruzada nas 2 dimensões, nomeadamente o item 10 e o item 11, cada um deles teve sempre uma saturação mais elevada na dimensão em que era suposto saturar. Ao efetuar-se a AFC obtiveram-se bons índices de ajustamento, nomeadamente $\mathrm{S}-\mathrm{B} \chi^{2} / \mathrm{df}=1,84 ; \mathrm{IFI}=0,99 ; \mathrm{CFI}=0,99 ; \operatorname{RMSEA}(90 \% \mathrm{CI})=0,07$ $(0,05-0,09)$. Dado que todos os itens cumpriram os critérios de nível de carga fatorial $\geq 0,50$, em termos de ACP e AFC, e de nível de correlação $\geq 0,30$, em termos de CITC, não foi necessário excluir itens (tabela 1 ).

0 passo seguinte consistiu em calcular a matriz de correlações, a consistência interna através de alfas de Cronbach e as médias das correlações interitens (tabela 2).

A validade convergente da NSSS-S (e suas dimensões) com a NSSS original (e suas dimensões) e com a SSSS demonstrou correlações positivas estatisticamente significativas, enquanto a validade divergente com a SBS demonstrou correlações negativas estatisticamente significativas. A validade concorrente com os itens ordinais de frequência de atividade sexual e de satisfação com a vida sexual demonstrou correlações positivas estatisticamente significativas (tabela 3).

A pontuação da NSSS-S e das suas dimensões foi de seguida distribuída por percentis, de forma a estabelecer pontos de referência para a sua utilização (tabela 4).

\section{Discussão}

O objetivo da presente investigação consistiu em analisar as propriedades psicométricas da NSSS-S. Procurou-se testar se a NSSS-S, tal como a NSSS original, teria uma estrutura bidimensional, tendo-se confirmado a existência dessa mesma estrutura bidimensional já detetada previamente ${ }^{11}$. Dois dos itens saturaram simultaneamente em ambas as dimensões, mas tal é entendível e até expectável, dado que ambas as dimensões são facetas do mesmo constructo de satisfação sexual. As correlações entre as dimensões da NSSS-S encontradas demonstraram ser altas e estatisticamente significativas, conforme esperado.

Em termos da fiabilidade, através do alfa de Cronbach verificou-se que tanto a escala total como as dimensões obtiveram valores bastante bons, sempre acima do valor de referência de 0,70 recomendado ${ }^{29}$. Todavia, no que 
+Model

ANDROL 1241-7

Tabela 1 Correlações item-total corrigidas e saturações da análise de componentes principais e da análise fatorial confirmatória

\begin{tabular}{llll}
\hline Itens da NSSS-S & CITC & Fator 1 ACP/AFC & Fator 2 ACP/AFC \\
\hline Item 2 & 0,71 & $0,72 / 0,77$ & $0,35 /-$ \\
Item 3 & 0,77 & $0,86 / 0,87$ & $0,29 /-$ \\
Item 5 & 0,79 & $0,83 / 0,88$ & $0,35 /-$ \\
Item 6 & 0,70 & $0,88 / 0,83$ & $0,19 /-$ \\
Item 8 & 0,67 & $0,68 / 0,75$ & $0,35 /-$ \\
Item 10 & 0,77 & $0,58 / 0,78$ & $0,54 /-$ \\
Item 11 & 0,77 & $0,50 /-$ & $0,66 / 0,83$ \\
Item 12 & 0,70 & $0,28 /-$ & $0,79 / 0,75$ \\
Item 14 & 0,65 & $0,20 /-$ & $0,82 / 0,74$ \\
Item 17 & 0,73 & $0,36 /-$ & $0,75 / 0,84$ \\
Item 19 & 0,76 & $0,44 /-$ & $0,70 / 0,85$ \\
Item 20 & 0,55 & $0,19 /-$ & $0,68 / 0,66$ \\
\hline
\end{tabular}

ACP: análise de componentes principais; AFC: análise fatorial confirmatória; CITC: correlações item-total corrigidas; NSSS-S: Nova Escala de Satisfação Sexual - versão curta.

Tabela 2 Matriz de correlações, alfas de Cronbach e médias das correlações interitens

\begin{tabular}{|c|c|c|c|c|c|}
\hline & NSSS-S & Subescala A & Subescala B & Alfa & MCII \\
\hline NSSS-S & 1 & & & 0,94 & 0,55 \\
\hline Subescala A & $0,93^{* * *}$ & 1 & & 0,92 & 0,64 \\
\hline Subescala B & $0,93^{\text {**** }}$ & $0,73^{* * *}$ & 1 & 0,89 & 0,58 \\
\hline
\end{tabular}

Alfa: alfa de Cronbach; MCII = médias das correlações interitens; NSSS-S=Nova Escala de Satisfação Sexual - versão curta; Subescala $A=$ centração no eu; Subescala $B=$ centração no parceiro e na atividade sexual.

*** significativo ao nível 0,001 .

Q9 Tabela 3 Validade convergente da Nova Escala de Satisfação Sexual - versão curta com a Nova Escala de Satisfação Sexual original e a Escala de Busca de Sensações Sexuais, validade divergente com a Escala de Aborrecimento Sexual e validade concorrente com as variáveis frequência de atividade sexual e satisfação com vida sexual

\begin{tabular}{lccc}
\hline & NSSS-S total & NSSS-S subescala A & NSSS-S subescala B \\
\hline NSSS total & $0,99^{* * * *}$ & $0,92^{* * * * *}$ & $0,92^{* * * *}$ \\
NSSS subescala A & $0,92^{* * *}$ & $0,99^{* * * *}$ & $0,73^{* * * *}$ \\
NSSS subescala B & $0,91^{* * *}$ & $0,73^{* * * *}$ & $0,97^{* * *}$ \\
SSSS & $0,20^{* * *}$ & $0,20^{* * *}$ & $0,16^{*}$ \\
SBS & $-0,43^{* * *}$ & $-0,43^{* * * *}$ & $-0,37^{* * *}$ \\
FAS & $0,20^{* * *}$ & $0,15^{* * *}$ & $0,22^{* * *}$ \\
SVS & $0,63^{* * *}$ & $0,54^{* * *}$ & $0,64^{* * *}$ \\
\hline
\end{tabular}

FAS: frequência de atividade sexual; NSSS: Nova Escala de Satisfação Sexual; NSSS-S: Nova Escala de Satisfação Sexual - versão curta; SBS: Escala de Aborrecimento Sexual; SSSS: Escala de Busca de Sensações Sexuais; Subescala A: centração no eu; Subescala B: centração no parceiro e na atividade sexual; SVS: satisfação com vida sexual.

*** significativo ao nível 0,001 ;

** significativo ao nível 0,01 ;

* significativo ao nível 0,05 .

Tabela 4 Percentis da Nova Escala de Satisfação Sexual - versão curta e suas dimensões

\begin{tabular}{llllllll}
\hline Percentis & 5 & 10 & 25 & 50 & 75 & 90 & 95 \\
\hline NSSS-S & 30,45 & 33 & 39 & 47 & 53 & 57,10 & 59 \\
NSSS-S A & 13,45 & 15 & 19 & 24 & 27 & 29 & 30 \\
NSSS-S B & 14 & 16 & 19 & 23 & 26 & 29 & 30 \\
\hline
\end{tabular}

NSSS-S: Nova Escala de Satisfação Sexual - versão curta; NSSS-S A: subescala de centração no eu; NSSS-S B: subescala de centração no parceiro e na atividade sexual. 
diz respeito às médias das correlações interitens a escala total e as dimensões obtiveram valores excessivamente elevados acima do valor de referência de 0,50 , indicando uma excessiva homogeneidade dos itens ${ }^{30}$. Relativamente às amplitudes de CITC obtiveram-se bons resultados quer considerando as dimensões isoladamente quer considerando a escala total dado que estiveram sempre acima do valor mínimo recomendado de $0,20^{31}$. De salientar que estes 2 últimos tipos de procedimentos de validação não foram efetuados originalmente na construção da escala.

Relativamente à validade convergente da NSSS-S efetuada com a NSSS original, evidenciou-se a existência de correlações positivas altas, que seria expectável, dado que ambas partilham em parte os mesmos itens. A validade convergente com a SSSS demonstrou correlações positivas moderadas-baixas e estatisticamente significativas, também esperadas, dado que teoricamente existe uma sobreposição considerável entre os constructos de satisfação sexual e de busca de sensações sexuais. $\mathrm{Na}$ validade divergente os resultados evidenciaram também bons valores, dado se ter comprovado as correlações negativas esperadas com a SBS devido aos constructos medidos serem concetualmente diferentes. A validade concorrente, feita com as variáveis frequência de atividade sexual e satisfação com vida sexual, demonstrou as esperadas correlações positivas e estatisticamente significativas ${ }^{32}$.

Em termos dos resultados obtidos pela presente investigação, devemos salientar algumas limitações. A utilização de uma amostra de conveniência exclusivamente urbana e com um nível de escolaridade superior originará alguma falta de representatividade dos nossos participantes face à população a nível nacional. Relativamente aos procedimentos técnicos de análise das propriedades psicométricas da NSSS-S, futuramente poder-se-á continuar o processo através de outros procedimentos complementares (e.g., estabilidade temporal, validade de grupos conhecidos) ${ }^{33}$.

\section{Conclusões}

É possível concluir que a análise das propriedades psicométricas da NSSS-S na população portuguesa revelou valores satisfatórios e muito semelhantes aos da NSSS original, inclusive a nível da estrutura fatorial bidimensional. Os investigadores e clínicos a trabalhar na área da sexualidade humana passam a ter à sua disposição um instrumento de autorresposta muito breve e devidamente adaptado para avaliar a satisfação sexual em homens e mulheres.

\section{Responsabilidades éticas}

Proteção de pessoas e animais. Os autores declaram que os procedimentos seguidos estavam de acordo com os regulamentos estabelecidos pelos responsáveis da Comissão de Investigação Clínica e Ética e de acordo com os da Associação Médica Mundial e da Declaração de Helsinki.

Confidencialidade dos dados. Os autores declaram ter seguido os protocolos do seu centro de trabalho acerca da publicação dos dados de pacientes.
Direito à privacidade e consentimento escrito. Os autores declaram ter recebido consentimento escrito dos pacientes e/ ou sujeitos mencionados no artigo. O autor para correspondência deve estar na posse deste documento.

\section{Financiamento}

A presente investigação foi parcialmente financiada pela Fundação para a Ciência e a Tecnologia (FCT) de Portugal.

\section{Conflito de interesses}

Os autores declaram não haver conflito de interesses.

\section{Anexo. Tradução portuguesa dos itens da} NSSS-S

Itens

2. A qualidade dos meus orgasmos.

3. A capacidade de me «soltar» e me entregar ao prazer sexual durante as relações.

5. A forma como eu reajo sexualmente ao(à) meu(minha) parceiro(a).

6. O funcionamento sexual do meu corpo.

8. 0 meu humor depois da atividade sexual.

10. O prazer que eu proporciono ao meu(minha) parceiro(a) sexual.

11. 0 equilíbrio entre o que eu dou e o que eu recebo durante o sexo.

12. 0 à-vontade do(a) meu(minha) parceiro(a) durante o sexo.

14. A capacidade do(a) meu(minha) parceiro(a) em ter orgasmos.

17. A criatividade sexual do(a) meu(minha) parceiro(a).

19. A diversidade das minhas atividades sexuais.

20. A frequência da minha atividade sexual.

NSSS-S: Nova Escala de Satisfação Sexual - versão curta.

\section{Referências}

1. Lawrance KA, Byers ES. Sexual satisfaction in long-term heterosexual relationships: The interpersonal exchange model of sexual satisfaction. Pers Relat. 1995;2(4):267-85.

2. Basson R. Biopsychosocial models of women's sexual response: Applications to management of desire disorders. Sex Relation Ther. 2003;18(1):107-15.

3. World Health Organization. (2010). Measuring sexual health: Conceptual and practical considerations and related indicators. Disponível em: http://whqlibdoc.who.int/hq/2010/ who_rhr_10.12_eng.pdf

4. Sánchez-Fuentes M, Santos-Iglesias P, Sierra JC. A systematic review of sexual satisfaction. Int $\mathrm{J}$ Clin $\mathrm{H}$ th Psyc. 2014;14(1):67-75.

5. Byers ES. Sexual satisfaction in romantic relationships: Findings from 25 years of research. Paper presented at the Conferência Sobre Satisfação Sexual, Faculdade de Psicologia da Universidade de Lisboa; 2011.

6. Sprecher S, Christopher FS, Cate R, Vangelisti AL, Perlman D. Sexuality in close relationships. In: Vangelisti A, Perlman D, 
editors. The Cambridge handbook of personal relationships. New York: Cambridge University Press; 2006. p. 463-82.

7. Stulhofer A, Busko V, Brouillard P. Development and bicultural validation of the new sexual satisfaction scale. J Sex Res. 2010;47(4):257-68.

8. Hudson WW, Harrison DF, Crosscup PC. A short-form scale to measure sexual discord in dyadic relationships. J Sex Res. 1981;17(2):157-74.

9. Rust J, Golombok S. The Golombok-Rust Inventory of Sexual Satisfaction (GRISS). Br J Clin Psychol. 1985;24:63-4.

10. Meston C, Trapnell P. Development and validation of a five-factor sexual satisfaction and distress scale for women: The sexual satisfaction scale for women (SSS-W). J Sex Med. 2005;2:66-81.

11. Mark KP, Herbenick D, Fortenberry JD, Sanders S, Reece M. A psychometric comparison of three scales and a single-item measure to assess sexual satisfaction. J Sex Res. 2014;51:159-69.

12. Pascoal PM, Narciso IB, Pereira NM. What is sexual satisfaction? Thematic analysis of lay people's definitions. J Sex Res. 2014;51:22-30.

13. Widaman KF, Little TD, Preacher KJ, Sawalani GM. On creating and using short forms of scales in secondary research. In: Trzesniewski KH, Donnellan MB, Lucas RE, editors. Secondary data analysis: An introduction for psychologists. Washington, DC: American Psychological Association; 2011. p. 39-61.

14. Stulhofer A, Busko V, Brouillard P. The New Sexual Satisfaction Scale and its Short Form. In: Fisher T, Davis C, Yarber W, Davis $\mathrm{S}$, editors. Handbook of Sexuality-Related Measures. $3^{\mathrm{a}}$ ed. New York: Routledge; 2011. p. 530-2.

15. Stulhofer A, Ferreira LC, Landripet I. Emotional intimacy, sexual desire, and sexual satisfaction among partnered heterosexual men. Sex Relation Ther. 2013;29(2):229-44.

16. Pascoal PM, Narciso I, Pereira NM. Emotional intimacy is the best predictor of sexual satisfaction of men and women with sexual arousal problems. Int J Impot Res. 2013;25(2):51-5.

17. Pechorro $P$, Calvinho A, Pereira N, Vieira R. Validação de uma versão portuguesa do Índice Internacional de Função Eréctil-5 (IIEF-5). Rev Int Androl. 2011;9(1):3-9.

18. Pechorro $P$, et al. Validação portuguesa da Nova Escala de Satisfação Sexual. Rev Int Androl. En prensa. 2015, http://dx.doi.org/10.1016/j.androl.2014.10.003.

19. Kalichman SC, Johnson JR, Adair V, Rompa D, Multhauf K, Kelly JA. Sexual sensation seeking: Scale development and predicting AIDS-risk behavior among homosexually active men. J Pers Assess. 1994;62(3):385-97.

20. Kalichman SC. Sexual Sensation Seeking Scale. In: Fisher TD, Davis CM, Yarber WL, Davis SL, editors. Handbook of Sexuality-Related Measures. $3^{\text {rd }}$ ed. New York: Routledge; 2011. p. 564-5.

21. Pechorro P, et al. Validação portuguesa da Escala de Busca de Sensações Sexuais. Rev Int Androl. 2015, http://dx.doi.org/ 10.1016/j.androl.2014.11.003. En prensa.

22. Watt JD, Ewing JE. Toward the development and validation of a measure of sexual boredom. J Sex Res. 1996;33: 57-66.

23. Pechorro $\mathrm{P}$, et al. Adaptação portuguesa da Escala de Aborrecimento Sexual. Rev Int Androl. 2015, http://dx.doi.org/ 10.1016/j.androl.2014.11.002. En prensa.

24. Hambleton R, Merenda P, Spielberger C. Adapting educational and psychological tests for cross-cultural assessment. Mahwah, NJ: Lawrence Erlbaum Associates; 2005.

25. IBM SPSS. IBM SPSS Statistics Base 22. Chicago, IL: SPSS Inc 2013.

26. Bentler P, Wu E. EQS for Windows user's guide. Encino, CA: Multivariate Software, Inc; 2008.

27. West S, Taylor A, Wu W. Model fit and model selection in structural equation modeling. In: Hoyle R, editor. Handbook of structural equation modeling. New York: The Guilford Press; 2012. p. 209-31.

28. Byrne B. Structural equation modeling with EQS: Basic concepts, applications, and programming. Mahwah, NJ: Lawrence Erlbaum Associates; 2006.

29. Nunnaly J, Bernstein I. Psychometric theory. New York: McGraw-Hill; 1994.

30. Clark L, Watson D. Constructing validity: Basic issues in objective scale development. Psychol Assess. 1995;7:309-19.

31. Marôco J. Análise estatística com o SPSS Statistics. Pero Pinheiro: ReportNumber; 2014.

32. Wiederman M. Reliability and validity of measurement. In: Wiederman $M$, Whitley $B$, editors. Handbook for conducting research on human sexuality. New Jersey: Lawrence Erlbaum Associates; 2002. p. 25-50.

33. Terwee CB, Bot SD, de Boer MR, van der Windt DA, Knol DL, Dekker J, et al. Quality criteria were proposed for measurement properties of health status questionnaires. J Clin Epidemiol. 2007;60:34-42. 